Trauma Berufskrankh 2017 · [Suppl 1]: 19:S112-S116 DOI 10.1007/s10039-016-0198-6

Online publiziert: 25. August 2016

๑) Springer-Verlag Berlin Heidelberg 2016

CrossMark

Ruprecht Beickert

BG-Unfallklinik Murnau, Murnau, Deutschland

\title{
Begutachtung des Abdominal- und Thoraxtraumas
}

zu unterziehen. Das heißt, er kann nicht unkritisch die Unfallfolgen des jeweiligen Fachgebietes in die Gesamtwertung übernehmen. Er sollte zunächst prüfen, ob die in der gesetzlichen Unfallversicherung geltenden Rechtsgrundlagen (und deren Terminologie) angewendet wurden, und er sollte nachsehen, ob die Funktionsstörungen nachvollziehbar gemessen und hinsichtlich der MdE korrekt eingeschätzt sind. Hierzu hilft ein Blick auf die in den einschlägigen Büchern gelisteten Erfahrungswerte. Die Ermittlung der Gesamt-MdE ist eine eigene Wertung, die den Stellenwert der Unfallfolgen und der daraus ableitbaren Teil-MdE der verschiedenen Fachgebiete würdigt.

\section{Fallbeispiel 1}

Ein 36-jähriger Arzt erlitt bei einem PKW-Unfall folgende Verletzungen:

- Brustkorbquetschung, Rippenserienfraktur 5-9 rechts,

- Lungenkontusion beidseitig, Lungenhämatom rechts,

- stumpfes Bauchtrauma, Mesenterial-, Jejunal- und Ileumruptur,

- Lisfranc-Luxationsfraktur links, Luxation Tarsometatarsal-Gelenke 4 und 5,

- Chopart-Luxation mit Fraktur des Os cuboideum,

- Talusluxationsfraktur rechts, Ruptur der Tibialis-posterior-Sehne,

- Innenband- und Außenbandruptur am Sprunggelenk.

Im Zuge der 6 Monate nach dem Unfall durchgeführten Untersuchung für das erste Rentengutachten wurden die nachstehenden Unfallfolgen festgestellt:
- Unfallchirurgisches Fachgebiet:

- Einschränkung Beweglichkeit rechts oberes Sprunggelenk (OSG) (15-0-15),

- Einschränkung Beweglichkeit rechts unteres Sprunggelenk (USG) (0-0-25),

- geringe Einschränkung der Zehenbeweglichkeit rechts,

- Umfangsvermehrung der rechten Knöchelregion bei Blutumlaufstörung,

- gering- bis mittelgradig gestörtes Gangbild,

- mittelgradige Belastungsminderung des rechten Beines,

- geringe Belastungsminderung des linken Beines,

- Verknöcherungen am rechten/ linken Beckenkamm (nach Einblutung),

- Hautgefühlsstörungen/Missempfindungen am rechten Fuß (N. tibialis),

- Hautgefühlsstörungen/Missempfindungen am linken Fuß (N. peroneus),

- geringe Deformierung des Lendenwirbelkörpers (LWK) 3;

- neurologisches Fachgebiet:

- leichte Parese der Zehenbeugung rechts,

- mittel- bis hochgradige Sensibilitätsminderung mit begleitenden Missempfindungen bei leichter Berührung rechts,

- leichtgradige Gefühlsminderungen im Bereich des Fußrückens links;

- internistisches Fachgebiet:

- Verlust des Endileums (ca. 30 cm), verminderte Resorption von $\mathrm{Vi}$ $\operatorname{tamin} \mathrm{B}_{12}$, Vitamin $\mathrm{D}_{3}$ und Gal- 


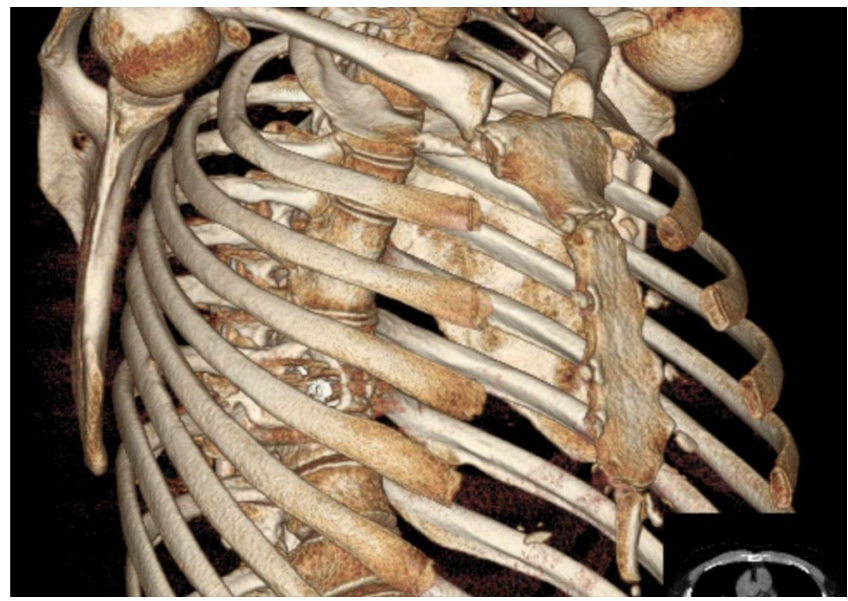

Abb. $1<$ Fallbeispiel 2: 3-D-Computertomographie eines Brustkorbes nach komplexer Thoraxwandverletzung mit Kompressionsfraktur des 8. Brustwirbels

lensäuren, dadurch Neigung zu Durchfällen.

- Die notwendigen Untersuchungen für die internistische Begutachtung sind:

- Sonographie des Bauchraumes,

- Laboruntersuchungen (Blutbild, Nieren- und Leberwerte),

- Magnetresonanztomographie (MRT; fakultativ bei chronischer Leberschädigung),

- MR-CP (Cholangiopankreatikographie) bei Gallenwegproblemen.

Im vorliegenden Fall führte der Verlust des Endileums zu einer Störung der Resorption von Vitamin $\mathrm{B}_{12}$. Der Blutspiegel des Vitamin $\mathrm{B}_{12}$ wird mit der Bestimmung des Holotranscobalamin im Serum gemessen.

Vitamin $B_{12}$ ist für die Zellteilung, Blutbildung und Nervenfunktion unerlässlich. Bei Vitaminmangel kann es zur perniziösen Anämie und zur funikulären Myelose kommen. Die funikuläre Myelose ist eine Rückenmarkerkrankung, bei der es zu Nervenausfallerscheinungen bis hin zur Querschnittlähmung kommen kann.

Da das Vitamin $B_{12}$ nicht mehr über das Endileum resorbiert werden kann, muss es parenteral verabreicht werden. In der akuten Mangelsituation werden die Körperspeicher zunächst aufgefüllt z. B. mit Hydroxocobalamin 4 Wochen lang $1000 \mathrm{ug} /$ Woche oder 1 bis 3 Wochen 2-mal $1000 \mathrm{ug} /$ Woche. Danach ist eine Erhaltungsdosis von 1000 ug alle 3 bis 6 Monate ausreichend.
Der Verlust des Endileums führt auch $\mathrm{zu}$ einer Resorptionsstörung der Gallensäuren, wodurch es zu wiederholten Durchfällen kommt.

Die MdE nach Verlust von Dünndarmanteilen wird wie folgt eingeschätzt [1]:

- $10 \%$ - mit geringen Auswirkungen,

- 20-30 \% - stärkere und häufig rezidivierende oder anhaltende Symptome (Durchfall, leichte Minderung des Ernährungszustandes),

- 40-50\% - erhebliche Minderung des Kräfte- und Ernährungszustandes, - bis $100 \%$ - bei Kurzdarmsyndrom.

In unserem Fallbeispiel wurde die internistische Teil-MdE mit 20 \% eingeschätzt.

\section{Milzverlust}

Der Verlust der Milz führt zu einer Schwächung der körpereigenen Abwehr und $\mathrm{zu}$ einer Vermehrung der Blutplättchen. Es liegt eine Störung der Filterfunktion und Ausreifung von bestimmten Zellen des Immunsystems (BZellen) vor.

Der Verletzte mit Milzverlust ist gefährdet, häufigere und schwerwiegendere Infektionen zu erleiden. Besonders empfänglich sind die Patienten für Infektionen mit Pneumokokken, Haemophilus influenzae und Meningokokken. Bei Milzverlust nach Trauma besteht ein 60fach höheres Infektionsrisiko. Besonders dramatisch ist die Postsplenektomiesepsis, deren Inzidenz mit 1,05-5\% und deren Mortalität mit $0,5 \%$. ermittelt wurden. Das Sepsisrisiko ist in den ersten Monaten nach dem Milzverlust sehr hoch und nimmt dann kontinuierlich in den Folgejahren ab, ohne jemals vollständig $\mathrm{zu}$ verschwinden. Deshalb sollten Versicherte mit Milzverlust bei fieberhaften Infektionen umgehend mit Antibiotika (Amoxicillin, Levofloxacin) behandelt werden. Die wichtigste Vorsorge gegen eine bedrohliche Infektion sind Impfungen.

Es gibt Impfstoffe gegen Pneumokokken (PSV-23/Pneumovax-23 ${ }^{\circledR}$ ), Haemophilus influenzae und Meningokokken.

Durch den Anstieg der Blutplättchen (Thrombozytose) besteht bei Asplenie ein erhöhtes Thromboserisiko. Besonders bedrohlich ist die Portalvenenthrombose $(2-5 \%)$, die v. a. in den ersten 2 Jahren nach dem Milzverlust auftritt. Eine Thromboseprophylaxe wird nicht durchgängig empfohlen. Sinnvoll ist die Einnahme eines Thrombozytenaggregationshemmers.

Die Minderung der Erwerbsfähigkeit nach Milzverlust wird wie folgt eingeschätzt [1]:

- $50-75 \%$ - 3. bis 6. Monat nach Milzverlust,

- 20-30\% - 6. bis 12. Monat nach

Milzverlust,

- 10-20\% - 13. bis 24. Monat nach

Milzverlust,

- $10 \%$ - auf Dauer.

\section{Thoraxtrauma}

Die notwendigen Untersuchungen für die Begutachtung des Thoraxtraumas sind:

- Lungenfunktionsanalyse in Ruhe und bei Belastung,

- Pleurasonographie, Zwerchfellbeweglichkeit,

- Thoraxröntgenaufnahme in 2 Ebenen fakultativ,

- Computertomographie (CT) des Thorax fakultativ (z. B. Aortendissektion).

Bei der Lungenfunktionsanalyse werden folgende Parameter ermittelt:

- Vitalkapazität (VC oder VK) maximales Luftvolumen, das ein oder ausgeatmet wird. 
- Einsekundenkapazität $\left(\mathrm{FEV}_{1}\right)$ Luftmenge, die in 1 Sekunde maximal ausgeatmet wird,

- Tiffeneau-Index ( $\left.\mathrm{FEV}_{1} / \mathrm{VC}\right)$ - Verhältnis der maximalen Ausatmung zum Einatmungsvolumen, Beurteilung der obstruktiven Atemwegserkrankung,

- Bodyplethysmographie:

- Residualvolumen (RV) - Luft, die nach dem Ausatmen in der Lunge verbleibt,

- Atemwegswiderstand (R) - Maß für den Widerstand der Atemwege, der z. B. bei Verengungen erhöht ist,

- Diffusionskapazität (DL) - Gasmenge pro Zeit, die von der Lunge aufgenommen wird.

Weitere Messparameter sind:

- inhalativer Provokationstest,

- Bronchospasmolysetest,

- Belastungsspirometrie,

- Peak-Flow-Meter.

Lungenfunktionsanalytisch werden

2 Hauptstörungen unterschieden:

- restriktive Ventilationsstörung: Diese liegt vor, wenn die Vitalkapazität verringert ist. Ursache ist die geringere Dehnbarkeit des Lungengewebes: es kann weniger Volumen eingeatmet werden;

- obstruktive Ventilationsstörung: Diese liegt vor, wenn die Einsekundenkapazität und das Residualvolumen erniedrigt sind. Ursache ist eine Einengung der Atemwege: Die Luft kann nur erschwert ausgeatmet werden, die Lunge überbläht (Asthma, „chronic obstructive pulmonary disease " [COPD]).

\section{Fallbeispiel 2}

Ein 28-jähriger in der Landwirtschaft Tätiger wird bei Baumfällarbeiten von einem Baumstamm am Brustkorb und Rücken getroffen. Es kommt zu folgenden Verletzungen:

- Brustwirbelkörper (BWK)-8-Fraktur,

- Querfortsatzfrakturen Brustwirbel

(BW) 3-11 links,

- Rippenserienfrakturen 1-9 rechts,
- Rippenserienfrakturen 6-7 links,

- Pneumothorax beidseitig,

- Pleuraergüsse beidseitig.

Ein Jahr nach dem Unfall werden nachstehende Unfallfolgen festgestellt:

- unfallchirurgisches Fachgebiet:

- Ersatz des 8. BWK durch einen Metallkörper,

- einliegender Fixateur externe über 3 Bewegungssegmente,

- Bewegungseinschränkung und Belastungsminderung der Brustwirbelsäule (BWS),

- Buckelbildung bei kyphotischer Knickbildung der mittleren BWS,

- plausibles Beschwerdebild;

- internistisches Fachgebiet:

- restriktive Lungenventilationsstörung mit Verminderung der Vitalkapazität um ein Drittel.

Bei der Nachuntersuchung 7 Jahre nach dem Unfall ergeben sich funktionell keine Änderungen (• Abb. 1, 2 und 3).

Die Minderung der Erwerbsfähigkeit ergibt sich aus den gemessenen Funktionsstörungen. Die Erfahrungswerte gehen von einer MdE von 20-50\% bei „Schwarten des Rippenfells mit Rippenverformungen oder Rippendefekten je nach Lungenfunktionsstörung und Brustkorbinstabilität“ aus [1].

Eine Lungenfunktionsstörung geringen Grades liegt vor, wenn die statischen und dynamischen Messwerte der Lungenfunktionsprüfung bis zu einem Drittel niedriger als die Sollwerte sind und die Blutgaswerte im Normbereich sind [2].

Eine Lungenfunktionsstörung mittleren Grades liegt vor, wenn die statischen und dynamischen Messwerte der Lungenfunktionsprüfung bis zu zwei Drittel niedriger als die Sollwerte sind und eine respiratorische Partialinsuffizienz vorliegt [2].

In unserem Fall lag eine geringgradige Lungenfunktionsstörung vor mit einer Teil-MdE des internistischen Fachgebietes von $20 \%$.
Trauma Berufskrankh 2017 . [Suppl 1]:

19:S112-S116

DOI 10.1007/s10039-016-0198-6

(c) Springer-Verlag Berlin Heidelberg 2016

\section{R. Beickert}

\section{Begutachtung des \\ Abdominal- und \\ Thoraxtraumas}

\section{Zusammenfassung}

Nach Arbeitsunfällen, die auch zu einer Thorax- und Bauchverletzung geführt haben, ist gutachterlich festzustellen, welche Unfallfolgen mit welchen funktionellen Ausfällen bestehen. Abgesehen von den chirurgisch zu bewertenden Unfallfolgen an der Brustkorbwand und an den Bauchdecken (inklusive Kunstafter) bleibt es dem internistischen Gutachter vorbehalten, die funktionellen Auswirkungen von Verletzungen der Brustorgane und/oder der Bauchorgane zu messen. Dem chirurgischen Gutachter obliegen dann die Zusammenfassung der Gutachten diverser Fachgebiete und die Ermittlung der GesamtMdE (Minderung der Erwerbsfähigkeit).

\section{Schlüsselwörter}

Gutachter · Funktionsausfall · Unfall . Minderung der Erwerbsfähigkeit .

Verletzung

\section{Expert assessment of abdominal and thoracic} trauma

\section{Abstract}

Following occupational accidents that also led to thoracic and abdominal injuries, it is necessary for experts to determine the presence of sequelae of injuries and any resulting functional deficits. Apart from the surgical assessment of the consequences of injuries of the chest wall and the abdominal wall (including colostomy), it is the responsibility of the internal medical expert to measure the functional effects of injuries of the thoracic organs and/or the abdominal organs. The surgical expert must then make a summary of the expert reports from various fields of expertise and determine the total loss of earning capacity.

Keywords

Expert opinion - Functional deficits . Accident - Loss of earning capacity · Injury 


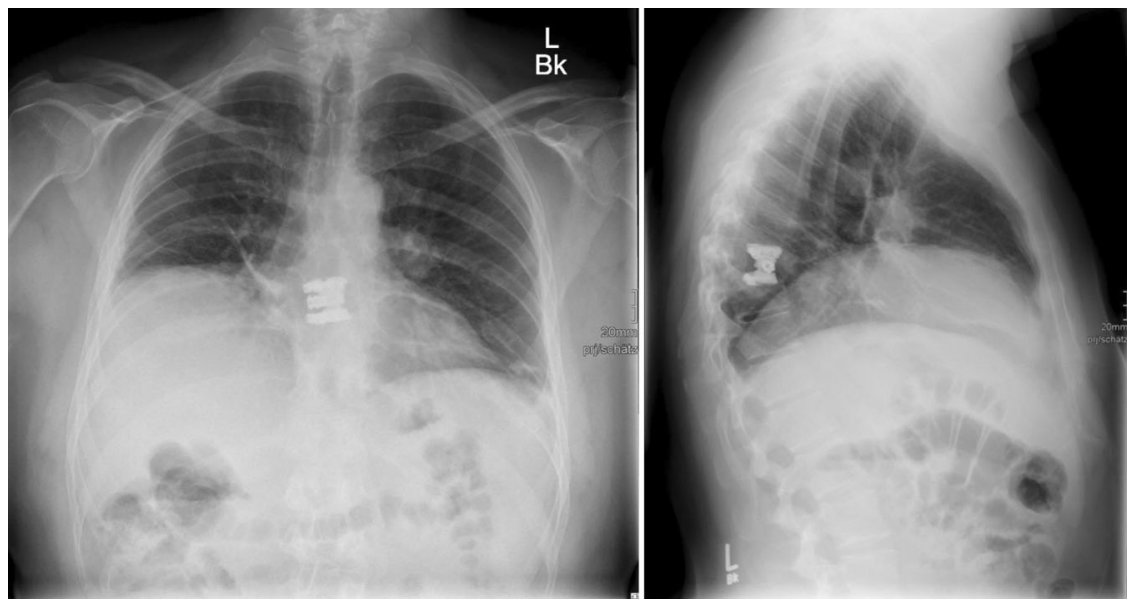

Abb. $2 \Delta$ Fallbeispiel 2: Thoraxröntgenaufnahme in 2 Ebenen. 8 Jahre nach komplexer Thoraxwandverletzung und Brustwirbelkörper (BWK)-8-Fraktur, kyphotische Knickbildung der Brustwirbelsäule (BWS), mangelhafte Inspiration bei Minderung der Vitalkapazität um ein Drittel

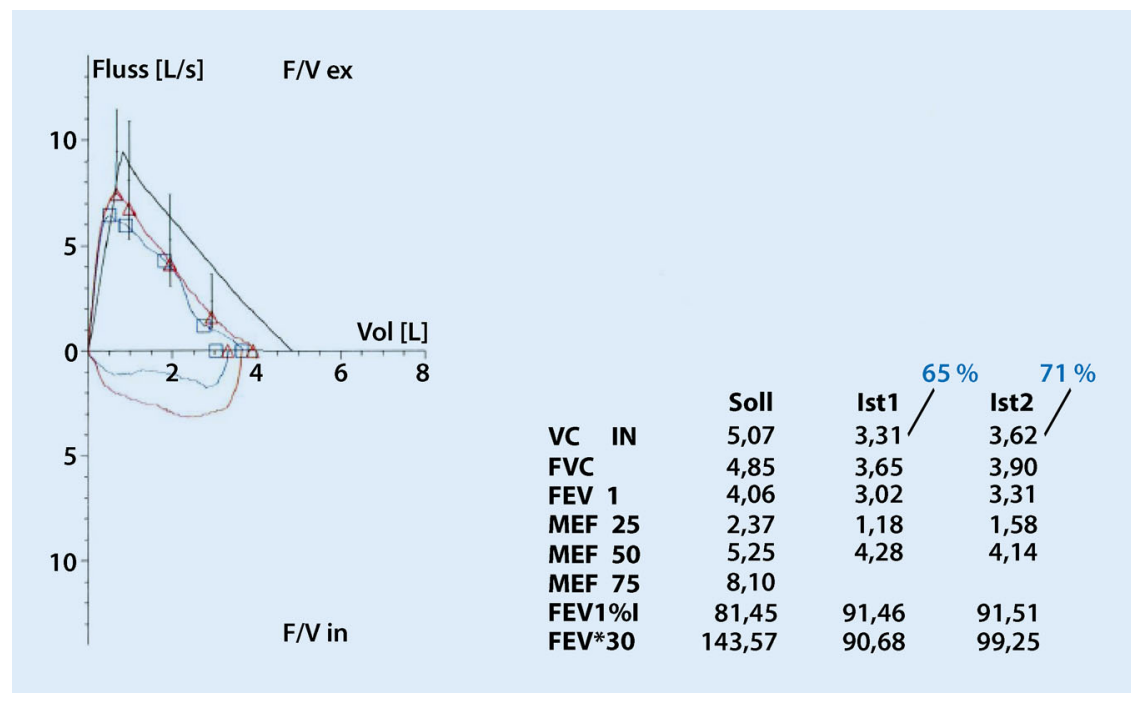

Abb. 3 A Fallbeispiel 2: Ergebnis der aktuellen Lungenfunktionsanalyse 7 Jahre nach dem Unfall (In Prozent angegeben: Einschränkung der Vitalkapazität im Vergleich zur Norm ist)

\section{Ermittlung der Gesamt-MdE}

Es gibt keine verbindlichen Regeln für die Ermittlung der Gesamt-MdE. Es gibt jedoch Grundsätze, die sich aus der Rechtsprechung entwickelt haben [3]:

- Das schematische Zusammenrechnen von Einzel-MdE verbietet sich (auch wenn Unfallfolgen sich nicht überschneiden).

- Es gilt, immer eine integrierende Gesamtschau aller Verletzungsfolgen vorzunehmen und deren Gesamtauswirkung auf die Erwerbsfähigkeit zu beurteilen. Vergleiche mit festen Eckwerten wie Amputationen oder
Nervenausfälle bei Rückenmarkverletzungen.

- Je höher eine Einzel-MdE in einem Fachgebiet ist, umso sorgfältiger ist zu prüfen, ob Funktionsstörungen aus anderen Fachgebieten, die mit einer niedrigen Einzel-MdE bewertet wurden, sich noch zusätzlich auf die verbliebenen Erwerbsmöglichkeiten auswirken [3].

Im praktischen Vorgehen ist zunächst für jedes verletzte Körperteil und jedes verletzte Organ unter funktionellen Gesichtspunkten eine „Teil-MdE“ zu ermitteln und festzuhalten. Maßstab hierfür sind die in der Gutachtenliteratur veröf- fentlichen Erfahrungswerte und Tabellen.

Dann ist von jedem Gutachter und integrierend - vom Hauptgutachter zu ermitteln und darzustellen, welche der ermittelten Funktionseinschränkungen

- ohne Überschneidung abgrenzbar sind,

- sich überschneiden,

- miteinander in Wechselwirkung stehen [1].

Die höchste der Einzel-MdE wird dann als Grundlage genommen, und die im Wert nachfolgenden werden daraufhin bewertet, wie durch sie die Gesamt-MdE beeinflusst wird.

\section{Fazit für die Praxis}

- Die Feststellung der Folgen und die Messung der Funktionsausfälle nach Verletzungen der Brust- und Bauchorgane obliegen in der Regel dem internistischen Gutachter.

- Die Messinstrumente sind die Lungenfunktionsanalyse, die Labordiagnostik, die Ultraschalluntersuchung des Brustkorbes und des Bauchraumes.

- Abzugrenzen sind Vorerkrankungen, Life-style-Faktoren wie Tabakkonsum oder Hyperalimentation sowie unspezifische Beschwerdebilder wie "Verwachsungsbeschwerden" und das Reizdarmsyndrom.

- MdE-relevant sind nur die objektiv messbaren Defekte und Funktionsausfälle.

\section{Korrespondenzadresse}

\section{Dr. R. Beickert}

BG-Unfallklinik Murnau

82418 Murnau, Deutschland

Ruprecht.Beickert@bgu-murnau.de

\section{Einhaltung ethischer Richtlinien}

Interessenkonflikt. R. Beickert gibt an, dass kein Interessenkonflikt besteht.

Dieser Beitrag beinhaltet keine vom Autor durchgeführten Studien an Menschen oder Tieren. 


\section{Übersichten}

The supplement containing this article is not sponsored by industry.

\section{Literatur}

1. Schönberger A, Mehrtens G, Valentin H (2010) Arbeitsunfall und Berufskrankheit. Erich Schmidt, Berlin

2. Bereiter-Hahn W, Mehrtens G (2016) Gesetzliche Unfallversicherung. Erich Schmidt, Berlin

3. Deutsche Gesetzliche Unfallversicherung (2015) Grundlagen der Begutachtung von Arbeitsunfällen 